\title{
Video display during laparoscopy - where should it be placed?
}

\author{
Dominik A. Walczak ${ }^{1,2}$, Dariusz Pawełczak², Piotr Piotrowski ${ }^{3}$, Piotr W. Trzeciak ${ }^{1}$, Adam Jędrzejczyk ${ }^{4}$, Zbigniew Pasieka² \\ ${ }^{1}$ Department of General Surgery, John Paul II Memorial Hospital, Belchatow, Poland \\ ${ }^{2}$ Department of Experimental Surgery, Medical University of Lodz, Lodz, Poland \\ ${ }^{3}$ Telecommunications and Teleinformatics Department, Technical University of Wroclaw, Wroclaw, Poland \\ ${ }^{4}$ Department of Urology, John Paul II Memorial Hospital, Belchatow, Poland
}

Videosurgery Miniinv 2015; 10 (1): 87-91

DOI: $10.5114 /$ wiitm.2014.47434

\begin{abstract}
Introduction: During laparoscopy, the monitor is usually placed near the operating table, at eye level, which significantly affects hand-eye coordination. First, it is impossible for the surgeon to simultaneously observe the operative field and hand movement. Second, the axis of view of the endoscope rarely matches the natural axis of the surgeon's sight: it resembles a direct view into the operative field. Finally, as the arms of the tools act as levers with a fulcrum at the site of the skin incision, the action of the tool handles is a mirror image of the movement of the tool tips seen on the monitor. Studies have shown that a neutral position with the head flexed at $15-45^{\circ}$ is the most ergonomically suitable.

Aim: To evaluate whether the level of monitor placement exerts an influence on laparoscopic performance.

Material and methods: A group of 52 students of medicine were asked to pass a thread through 9 holes of different sizes, placed at different levels and angles, using a self-made laparoscopic simulator. Each student performed the task four times in two monitor positions: at eye level, and placed on a simulator. The order of monitor placement was randomized.

Results: The task was performed more quickly when the monitor was placed on the simulator and the sight was forced downwards. Lower placement was also found to be more beneficial for students with experience in laparoscopy. Conclusions: New technologies which place the display on the patient, thus improving the ergonomics of the operation, should be developed.
\end{abstract}

Key words: laparoscopy, surgical training, monitor, video display, ergonomics.

\section{Introduction}

During laparoscopic procedures, the image is usually displayed on a single LCD monitor placed on an instrument tower at eye level, near the operating table. However, this mode of visualization has many limitations. Firstly, a three dimensional reality is displayed on a two-dimensional screen, with a consequent loss of depth perception. Second, the axis of view of the endoscope rarely matches the natural axis of the surgeon's sight, giving the impression that the surgeon is looking directly into the operative field. Hence, it is impossible for the surgeon to simultaneously observe both the operative field and hand movement, which significantly affects hand-eye coordination. Finally, as the arms of the tools act as levers with a fulcrum at the site of the skin incision, the real action with tool handles is a mirror image of the movement of the tool tips seen on the monitor [1].

The consequence of such monitor placement is a forced twisting of the neck and body and slight el-

\section{Address for correspondence}

Dominik A. Walczak MD, Department of General Surgery, John Paul II Memorial Hospital, 123 Czalpiniecka St, 97-400 Belchatow, Poland, phone: +48 604436 868, e-mail: dr.dominikwalczak@gmail.com 
evation of the head, resulting in faster fatigue of the muscles. Studies have shown that a neutral position with the head flexed at $15-45^{\circ}$ is the most comfortable. What is more, looking down improves accommodation of the eye lens, resulting in less eye weariness and fewer headache episodes in the surgeon [2-4].

It is important to mention that a minimally invasive procedure is usually longer and the procedure more strenuous for the operating team than in conventional surgery. Therefore, it is important to provide conditions which are more ergonomically suitable for the surgeon in future operation rooms, which includes optimizing the monitor position.

\section{Material and methods}

Fifty-two medical students (32 males, 12 females) participated in this study, with an average age of 23.3 years $(S D=2.26)$. Twenty-four group members were novices with no prior experience in performing this type of surgery. However, the remaining 28 students had participated in a 2-hour laparoscopic course organized by another surgical department 6 months earlier, but with no more experience in laparoscopic surgery. All participants had 20/20 normal or corrected vision and were compensated for their time.
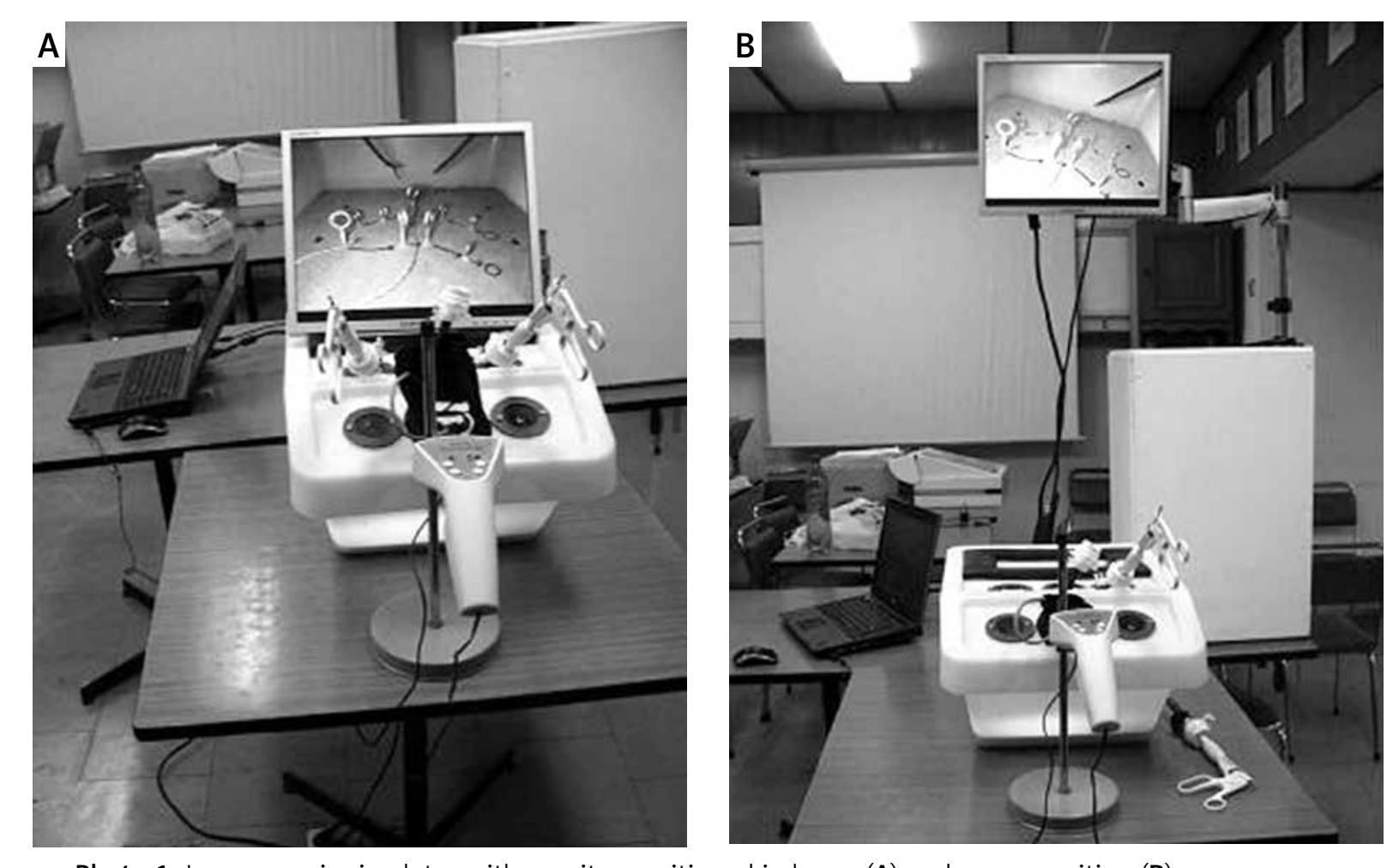

Photo 1. Laparoscopic simulator with monitor positioned in lower (A) and upper position (B)
A laparoscopic simulator was designed and created for the purposes of the study. The housing was a small 'Sortera' plastic box with a fitted lid (Ikea). The plastic wall is $2 \mathrm{~mm}$ thick and rigid, which is crucial for the stability of the construction. Six holes were cut from the lid. In five holes, pieces of rubber sheet were placed to imitate the elasticity of the integument. The middle (largest) aperture was intended for camera placement. A Logitec Quickcam pro 9000 webcam was used as the camera, and six LEDs were placed on its casing to act as a light source; this ensured that the axis of view of the camera rarely matched the illumination axis and no shadows were present.

A detailed description of the laparoscopic simulator with additional photos and advice for future constructors was published previously [5].

A 17-inch LCD monitor with a $4: 3$ aspect ratio was used during the experiment. Monitors currently used in actual operating rooms have similar characteristics. Two monitor placement conditions were tested: in the first, the monitor was positioned on the simulator $20^{\circ}$ below eye level and $0.6 \mathrm{~m}$ away from the participant (Photo $1 \mathrm{~A}$ ); in the second, the monitor was placed at a height of $1.60 \mathrm{~m}$ from the 
floor to the base of the monitor, $1 \mathrm{~m}$ away from the participant, similar to conditions in our operating room (Photo $1 \mathrm{~B}$ ).

Before the task, a 5-minute introductory exercise was performed to allow the students to became familiar with the simulator. Using laparoscopic graspers, the participant lifted wooden matches one by one from the middle of the board, transferring the object in midair to the second grasper, and then placing it on a plastic plate - once with their dominant and once with their non-dominant hand.

The proper task was to pass a thread through 9 holes. For this purpose, a pad was created using medium-density fibreboard and 9 eye bolts. There were two sizes of aperture: larger (4 pcs) $6 \mathrm{~mm}$ diameter and smaller (5 pcs) $4.5 \mathrm{~mm}$. The eyes were placed at different levels (1-4 cm) and angles (Photo 2) The green thread was made from polyamide: it was $20 \mathrm{~cm}$ long, $2 \mathrm{~mm}$ thick, and both ends were flamed to prevent single filaments from being untangled. The task was performed using two $5 \mathrm{~mm}$ serrated graspers. Each student performed the task four times in two monitor positions: once at eye level, and once placed on the laparoscopic simulator. The order of monitor placement was randomized. At the end of the study, the students were asked which position they preferred.

\section{Statistical analysis}

Statistical analysis was undertaken using the Mann-Whitney $U$ test, with $p<0.05$ being taken as significant.

\section{Results}

The best time for a single attempt was $118 \mathrm{~s}$, while the worst was $935 \mathrm{~s}$. The average time for par-

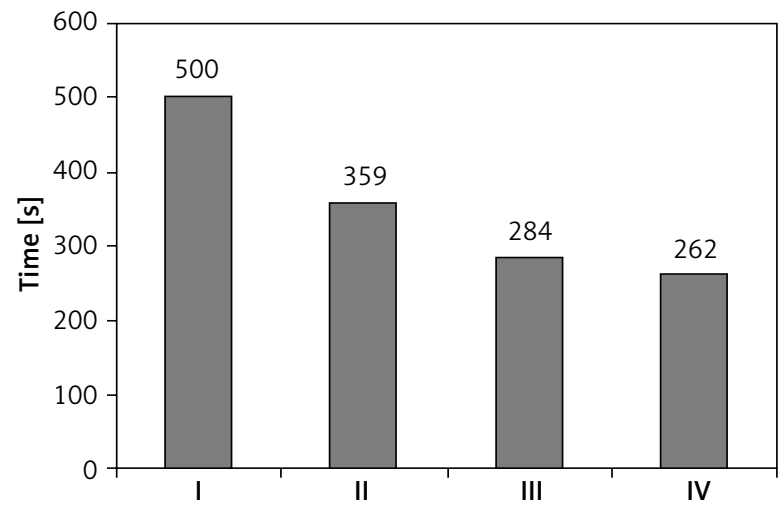

Figure 1. Average time for particular attempts

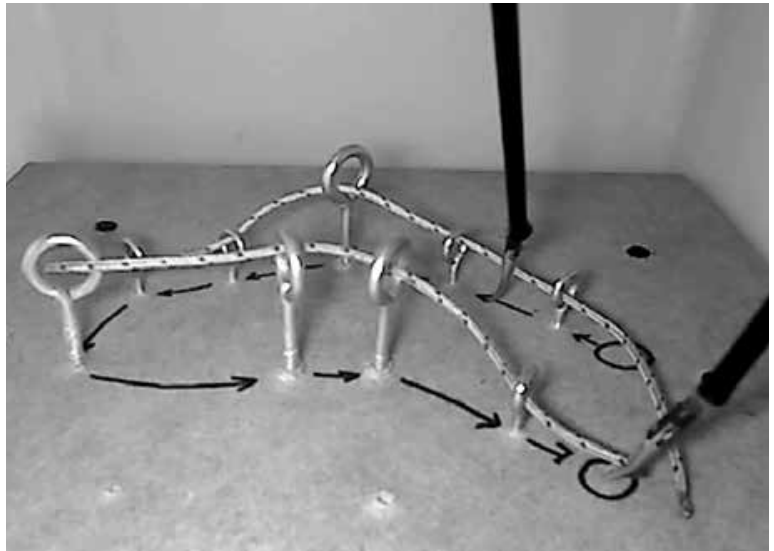

Photo 2. Task

ticular attempts improved, irrespective of the monitor position (Figure 1). The average times for the first and second tries were significantly better $(p=0.04)$ when the monitor was placed in the lower position (376 s) than in the upper position (476 s). The times for the third and fourth tries were also shorter when the video display was positioned on the simulator (283s vs. 258 s), but not significantly so.

Students who had attended the laparoscopy course performed the task more quickly than novices (Table I). Moreover, students with some experience in laparoscopy also performed the task faster when the monitor was placed at hand level. In this subgroup, the average time for the first and second tries was $301 \mathrm{~s}$ when the monitor was placed in the lower position and $360 \mathrm{~s}$ in the upper position $(p=0.12)$. The results for the third and fourth attempts were better when the video display was positioned on the simulator (200 s vs. 262 s, $p=0.04$ ). A slightly greater proportion of students preferred the lower to the upper position: $56 \%$ vs. $44 \%$, respectively.

Table I. Average time for particular attempts for novice students with no prior experience in laparoscopic surgery and students after a 2-hour laparoscopic course

\begin{tabular}{|lccc|}
\hline Attempt & $\begin{array}{c}\text { Time taken by } \\
\text { novice students } \\
{[\mathrm{s}]}\end{array}$ & $\begin{array}{c}\text { Time taken } \\
\text { by non-novice } \\
\text { students [s] }\end{array}$ & $\begin{array}{c}\text { Value } \\
\text { of } p\end{array}$ \\
\hline I & 667 & 357 & 0.01 \\
\hline II & 429 & 303 & 0.02 \\
\hline III & 335 & 244 & 0.02 \\
\hline IV & 314 & 222 & 0.02 \\
\hline
\end{tabular}




\section{Discussion}

According to the results of the present study, the time taken to perform the task was shorter when the monitor was placed on the simulator and the sight was forced downwards. The benefits of this lower placement were also observed when students with some experience in laparoscopy performed the task.

Although few studies have attempted to determine the best position for the monitor during laparoscopy, the existing data confirm our results. When evaluating the quality of surgical knots tied in a laparoscopic trainer at varying monitor locations, Hanna et al. note that the best results were observed when the video display was placed in front of the operator at the level of the manipulation workspace (hands). This configuration permitted the operator to operate the trainer by gaze-down viewing, and the alignment of the visual and motor axis was similar to conventional operation [6].

Omar et al. confirmed that many benefits exist for such positioning in even more complicated tasks by changing the position of the endoscope to obtain $45^{\circ}$ and $90^{\circ}$ divergence between the image axis and the axis of manipulations performed in the simulator. It is well known that adaptation to the inverted picture needs much greater mental involvement, as can be demonstrated by increased brain cortex activity, resulting in a higher number of mistakes and longer duration for the procedure. The findings show that directing the eyes downward reduces the discomfort associated with these geometrical transformations and facilitates more successful task completion [1, 7-9].

The placement of the video display on the patient's abdomen seems to be the perfect solution. However, the bulk of the monitor, the limited quality of the image seen from such an angle and the need for a sterile cover detract from this approach. Projecting the image onto a light-weight, sterile screen directly above the patient's body might be an alternative solution. An additional benefit of such a presentation method is lack of the casing which always encloses classic LCD monitors. The frame generates a psychological cue that the image is only two-dimensional [1, 10, 11].

For a laparoscopic bowel-suturing task, Brown et al. do not note any difference with respect to surgical task performance measures (completion time, leakage pressure, and error score) or physical discomfort (visual strain, headache, and neck/shoulder pain) between using a monitor placed at approximately eye level or a video projection system projecting the image onto a screen placed close to the hands. The authors state that performance of the projector suffered due to the low quality of the image, which outweighed the potential advantages of the gaze-down stance. Only the use of modern projectors and with image quality equal or superior to modern monitors would confirm the benefits of such positioning [12].

Perhaps modern visualization methods may allow the benefits from gaze-down viewing to be incorporated into laparoscopic surgery. One concept of imaging utilizes structural light and augmented reality (AR). Augmented reality can be understood as a compromise between virtual reality (VR), i.e. completely artificial, computer-generated images, and telepresence, i.e. true, overlaid images. In contrast to VR, where an operator is completely "submerged" in a fake environment, the user of augmented reality can see the surrounding, real world with virtual objects overlaying it. Thus, AR contributes to the reality instead of substituting for it. Virtual and real objects coexist in the same space, creating an effect which may be seen in the movie Who Framed Roger Rabbit? [1, 13].

In this technology, a special projector emits light beams called high frequency rasters, which form linear illuminations on the surface of encountered objects. Observing this striatum at the proper angle and analysing the visible deformity provides 3-dimensional information. With this technology, Chapel Hill scientists have created a 3D endoscope. They used a standard laparoscope as a structural light source. A video camera mounted alongside the metal tube facilitated observation of the pattern of bands and transmission of information to the computer for further analysis. The image was presented to the surgeon with a head-mounted optical display. The operator may view the internal organs through a virtually generated window on the skin, showing organs and tissues in real time. This innovative system combines advantages of 3D imaging and gazedown viewing. However, it has not yet been used in humans, and all experiments have been performed on phantoms only $[1,14]$.

\section{Conclusions}

Our studies show that the benefits from lower placement of the monitor were gained by both nov- 
ice students and those with a little experience in laparoscopy. New technologies which place the display on the patient should be developed, as these will improve the working position of the surgeon and improve efficiency.

\section{Conflict of interest}

The authors declare no conflict of interest.

\section{References}

1. Grobelski B, Walczak DA, Pasieka Z. New ways of visualization in laparoscopic surgery. Videosurgery Miniinv 2010; 5: 120-8.

2. Matern U, Faist M, Kehl K, Giebmeyer C. Monitor position in laparoscopic surgery. Surg Endosc 2005; 19: 436-40.

3. Burgess-Limerick R, Mon-Williams M, Coppard VL. Visual dis play height. Hum Factors 2000; 42: 140-50.

4. Cuschieri A. Epistemology of visual imaging in endoscopic surgery. Surg Endosc 2006; Suppl. 2: S419-24.

5. Walczak DA, Piotrowski P Jędrzejczyk A, et al. A laparoscopic simulator - maybe it is worth doing it yourself? Videosurgery Miniinv 2014; 9: 380-6.

6. Hanna GB, Shimi SM, Cuschieri A. Task performance in endoscopic surgery is influenced by location of the image display. Ann Surg 1998; 227: 481-4.

7. Omar AM, Wade NJ, Brown SI, Cuschieri A. Assessing the benefits of "gaze-down" display location in complex tasks. Surg Endosc 2005; 19: 105-8.

8. Ittelson WH. Mirror reversals: real and perceived. Perception 1993; 22: 855-61.

9. Bock O, Abeele S, Eversheim U. Human adaptation to rotated vision: interplay of a continuous and a discrete process. Exp Brain Res 2003; 152: 528-32.

10. Cuschieri A. Visual display technology for endoscopic surgery. Minim Invasive Ther Allied Technol 1996; 5: 427-34.

11. Wade NJ. Frames of references in vision. Minim Invasive Ther Allied Technol 1996; 5: 435-9.

12. Brown SI, Frank TG, El Shallaly G, Cuschieri A. Comparison of conventional and gaze-down imaging in laparoscopic task performance. Surg Endosc 2003; 17: 586-90.

13. Azuma R. A survey of augmented reality. Presence: Teleoperators and Virtual Environments 1997; 6: 355-85.

14. Fuchs H, Livingston MA, Raskar R, Colucci D. Augmented reality visualization for laparoscopic surgery. Lect Notes Comput Sci 1998; 1496: 934-43.

Received: 20.10.2014, accepted: 26.10.2014 